\title{
Optimization of Hybrid Energy Storage System Control Strategy for Pure Electric Vehicle Based on Typical Driving Cycle
}

\author{
Kanglong Ye, ${ }^{1}$ Peiqing Li $\mathbb{D},{ }^{1,2,3}$ and $\mathrm{Hao}^{\mathrm{Li}}{ }^{1}$ \\ ${ }^{1}$ School of Mechanical and Energy Engineering, Zhejiang University of Science and Technology, Hangzhou 310023, China \\ ${ }^{2}$ School of Mechanical Engineering, Zhejiang University, Hangzhou 310058, China \\ ${ }^{3}$ Zhejiang Fuchunjiang Communication Group, Hangzhou 310089, China \\ Correspondence should be addressed to Peiqing Li; lpqing@hotmail.com
}

Received 31 January 2020; Revised 24 May 2020; Accepted 27 May 2020; Published 29 June 2020

Academic Editor: Haopeng Zhang

Copyright ( $\odot 2020$ Kanglong Ye et al. This is an open access article distributed under the Creative Commons Attribution License, which permits unrestricted use, distribution, and reproduction in any medium, provided the original work is properly cited.

Taking a hybrid energy storage system (HESS) composed of a battery and an ultracapacitor as the study object, this paper studies the energy management strategy (EMS) and optimization method of the hybrid energy storage system in the energy management and control strategy of a pure electric vehicle (EV) for typical driving cycles. The structure and component model of the HESS are constructed. According to the fuzzy control strategy, aimed at the roughness of the membership function in EMS, optimization strategies based on a genetic algorithm (GA) and particle swarm optimization (PSO) are proposed; these use energy consumption as their optimal objective function. Based on the improved EV model, the fuzzy control strategy is studied in MATLAB/Advisor, and two control strategies are obtained. Compared with the simulation results based on three driving cycles, urban dynamometer driving schedule (UDDS), new European driving cycle (NEDC), and ChinaCity, the optimum control strategy were obtained. The theoretical minimum energy consumption of HESS was reached by dynamic programming (DP) algorithm in the same simulation environment. The research shows that, compared with the PSO, the output current peak and current fluctuation of the battery optimized by the GA are lower and more stable, and the total energy consumption is reduced by 3-9\% in various simulation case studies. Compared with the theoretical minimum value, the deviation of energy consumption simulated by GA-Fuzzy Control is $0.6 \%$.

\section{Introduction}

In recent years, new energy vehicles have become the main development direction of the automobile industry. Compared with fuel vehicles, pure electric vehicles have the characteristics of energy saving and environmental protection without exhaust pollution. However, owing to the influence of the battery material, power supply form, power management strategy, and driving environment, pure electric vehicles (EVs) have defects such as short endurance mileage and poor energy consumption stability. Among them, whether the energy management and control system of a hybrid energy storage system (HESS) can provide the best energy distribution strategy according to changes in the working conditions has become one of the hot spots in current research on the energy management and control of
EVs. By improving the power management and control system of electric vehicles, the stability of the management strategy and endurance mileage of EVs can be improved.

To improve the energy control efficiency of EVs and emphasize real-time optimization, domestic and overseas scholars have conducted significant amounts of research on energy control strategies and predictive control. Some scholars optimized the working efficiency of the power system by improving the components of the HESS. In $[1,2]$, a new hybrid battery/ultracapacitor energy storage system for electric vehicles (including electric vehicles, hybrid vehicles, and plug-in hybrid vehicles) was proposed. This system uses a smaller DC/DC converter as a controlled energy pump to keep the voltage of the ultracapacitor higher than that of the battery under urban driving conditions. In [3], a modulator replaces the DC/DC converter for 
connections, which solves the problem of large voltage changes caused by ultracapacitor power transmission. Thus, the drive performance of the motor is not disturbed.

Research on energy management strategies can optimize the energy efficiency of the entire vehicle without changing the basic components and framework of the HESS. In [4], an optimization framework was proposed to calculate the suboptimal current of the hybrid system in an EV to control the current and minimize the working current and fluctuation of the battery in the EV. In [5-8], by combining a battery and ultracapacitor, an energy control strategy with fuzzy control strategy as the core was proposed to improve the fuel economy and durability of energy system components while maintaining the vehicle power performance. In $[9,10]$, a real-time optimal energy management strategy (EMS) was proposed for a plug-in hybrid bus based on the minimum equivalent fuel consumption strategy and considering the frequent starting of a low-speed engine. In $[11,12]$, the energy management of a hybrid power system was analyzed. The energy flow of different energy sources was managed separately by combining fuzzy logic control and shape control. In [13], the online predictive control strategy of a series and parallel plug-in hybrid EV was studied. A new dual-loop online intelligent planning (DOIP) method for speed prediction and energy flow control was proposed, and a depth fuzzy predictor was established to realize directional speed prediction. The optimal control behavior was determined by learning the vehicle speed and acceleration.

There are also some scholars who use algorithms to optimize research. Reference [14] has proposed an adaptive power distribution algorithm. The parameters of the algorithm were optimized by combining self-organizing mapping and particle swarm optimization (PSO) to alleviate the peak demand and short charge and discharge period of the battery. In $[15,16]$, the focus is on the $k$-means clustering algorithm. Reference [15] focuses on the study of the driving cycle of cars in Tehran and its suburbs. By collecting the situation of vehicles running in actual traffic, the calculation is based on the definition of "micro-travel." Two driving functions, "average speed" and "idle time percentage," are calculated. The micro-trips are then clustered into four groups in driving feature space using the $k$-means clustering method. The focus of [16] is the application of driving condition recognition in hybrid electric vehicle intelligent control. For this purpose, driving features are identified and used for driving segment clustering, using the $k$-means clustering algorithm. Many combinations of driving features and different numbers of clusters are evaluated, in order to achieve the best traffic condition recognition results. References $[17,18]$ also record the vehicle data and driving data under the actual traffic conditions, analyze the driving end, and study the influence of driving characteristics on the fuel consumption and exhaust emissions through the driving segment simulation. The results of [17] show that the velocity-dependent driving features such as "energy," "mean of velocity," "displacement," and "maximum velocity" are more effective in vehicle exhaust emissions and fuel economy. The results of [18] show that "energy" and "percentage of free time" are two driving characteristics to drive segmented clustering. Driving segment clustering can be used in driving cycle development, intelligent hybrid vehicle control and so on.

In the current study, most of the control strategies of EVs are optimized in one algorithm. Based on the above research, this paper uses fuzzy control strategy as an EMS with the composite power supply form of a combined battery and ultracapacitor. Reducing the total energy consumption is the optimization goal, and GA and PSO are used to carry out learning optimization for the fuzzy control strategy in MATLAB/Advisor in the UDDS, NEDC, and ChinaCity driving cycles. In the same simulation environment, the theoretical minimum energy consumption of HESS is calculated by DP algorithm. An experimental analysis is carried out to compare the battery current output performance and the total energy consumption parameters of the energy system and to evaluate the optimization effect of the algorithm.

\section{System Description and Methodology}

2.1. Modeling of HESS. There are three common types of composite power supply structure: passive, semiactive, and active. The semiactive structure has two types: battery end load and capacitor end load. The main feature of the passive hybrid energy system is that the battery and the ultracapacitor are directly connected in parallel on the power bus, and the voltage of the two is synchronized in real time such that the energy distribution between the battery and ultracapacitor cannot be flexibly adjusted in the case of highpower output or full capacity. The composite power supply with an active structure has two DC/DC converters that are connected in series with batteries and capacitors. The two DC/DC converters are integrated and connected in series. Both of these forms can flexibly adjust the distribution of the energy output, but the energy system structure is complex and the actual application cost is high [19].

Therefore, in this study, the battery terminal load structure of a semiactive energy system is selected. Its topology is shown in Figure 1. The battery and capacitor are isolated by a DC/DC converter. The battery acts as the main power supply, and the capacitor is the auxiliary power supply. The energy system distribution of the battery and capacitor can be realized through high and low voltage conversions of the voltage. Compared with the battery end load, the ultracapacitor is used as the main power supply in the form of the capacitor end load. Owing to the low capacity of the capacitor energy, the battery needs to participate in driving, and all current flowing through the battery needs to pass through the DC/DC converter. This makes the battery work with low efficiency and high energy consumption.

2.2. Modeling of Battery. Battery modeling is a significant task within battery technology development and is vital in applications. For example, EV range prediction is only possible through the application of advanced battery modeling and estimation techniques to determine current 


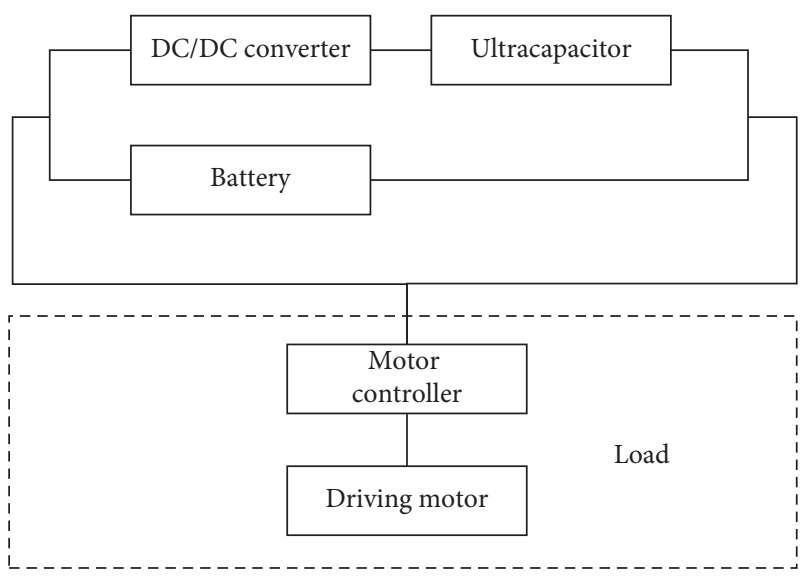

Figure 1: Model of the vehicle electrical energy system.

state and predict remaining endurance. In addition, battery modeling is essential for safe charging and discharging, optimal utilization of batteries, fast charging, and other applications [20].

A battery simulation model verifies the correctness of the model parameter settings in a simulation. Generally, the input is the current, and the output is the terminal voltage. Because the current, power, temperature, state of charge (SOC), and other parameters have nonlinear effects on the battery characteristics, considering all factors in modeling makes the simulation calculation too large and difficult to control.

Equivalent circuit models of a battery include the Rint model, the Thevenin model, and the second-order reserve capacity (RC) model. The Rint model is the equivalent circuit model of internal resistance, which regards the battery as a series model of the ideal voltage source and resistance. In this model, it is easy to set the parameters and run a simulation, but the accuracy is low. The Thevenin model is a first-order RC model and contains a voltage source and an RC parallel circuit. The model fully considers the relationship between the electromotive force and SOC and the dynamic process of the battery. It can accurately simulate the battery charging and discharging process, but it does not consider the open-circuit voltage changes caused by the current accumulation, so it is not suitable for long-time simulations. The second-order RC model adds a group of RC circuits on the basis of the Thevenin model. In the model, the variable voltage source connects the resistance and two RC circuits. This can provide better consideration to the transient and steady-state characteristics of the battery but does not consider the influence of the temperature and battery self-discharge [21, 22].

This paper compares the optimization effects of different algorithms and does not require a high-precision simulation, so the more universal Rint model is selected for modeling. Figure 2 shows the battery equivalent circuit model, and the mathematical model is described as follows:

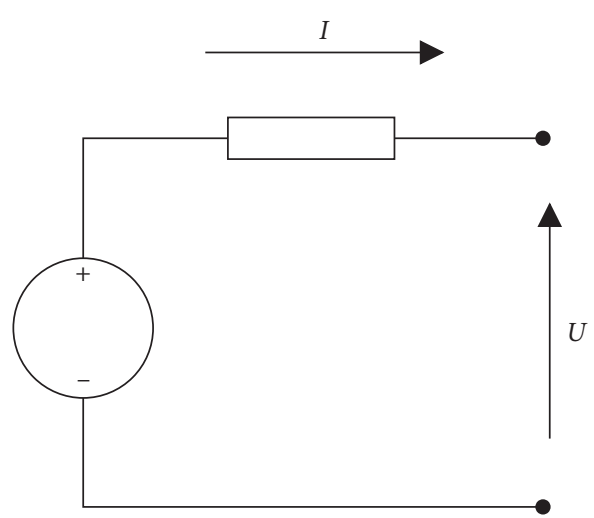

FIGURE 2: Internal-resistance model of the battery.

$$
\left\{\begin{array}{l}
Q_{b}=n_{b 1} n_{b 2} Q_{\mathrm{bc}} \\
R_{b}=\frac{n_{b 1} R_{\mathrm{bc}}}{n_{b 2}} \\
U_{b}=n_{b 1} U_{\mathrm{bc}} \\
\frac{\mathrm{dSOC}}{\mathrm{d} t}=\frac{U_{b}-\sqrt{U_{b}^{2}-4 R_{b} P_{m}}}{2 R_{b} Q_{b}}, \\
P_{b}=-\frac{\mathrm{dSOC}}{\mathrm{d} t} U_{b} Q_{b},
\end{array}\right.
$$

where $Q_{b}, R_{b}$, and $U_{b}$, respectively, represent the capacity, internal resistance, and terminal voltage of the battery pack; $Q_{\mathrm{bc}}, R_{\mathrm{bc}}$, and $U_{\mathrm{bc}}$, respectively, represent the capacity, internal resistance, and terminal voltage of the battery cell; $n_{b 1}$ and $n_{b 2}$, respectively, represent the number of series and parallel modules in the battery pack; and $P_{b}$ is the power density of the battery.

2.3. Modeling of Ultracapacitor. An ultracapacitor is a type of electrochemical element that stores energy by virtue of physical characteristics. Unlike a battery with a large capacity, the ultracapacitor has a higher energy density, higher charge and discharge power, and longer cycle life. It is suitable to use for power transport in the start and stop stages, active suspension systems, and rapid acceleration stage. In recent years, ultracapacitors have been widely used in high-power energy storage systems of vehicles, ships, and aerospace projects [23-25].

The RC internal-resistance model, which is common and easy to implement, is also selected to describe the ultracapacitor. The model is generally composed of a series resistance, parallel resistance, and ideal capacitor. The equivalent circuit model is shown in Figure 3. 


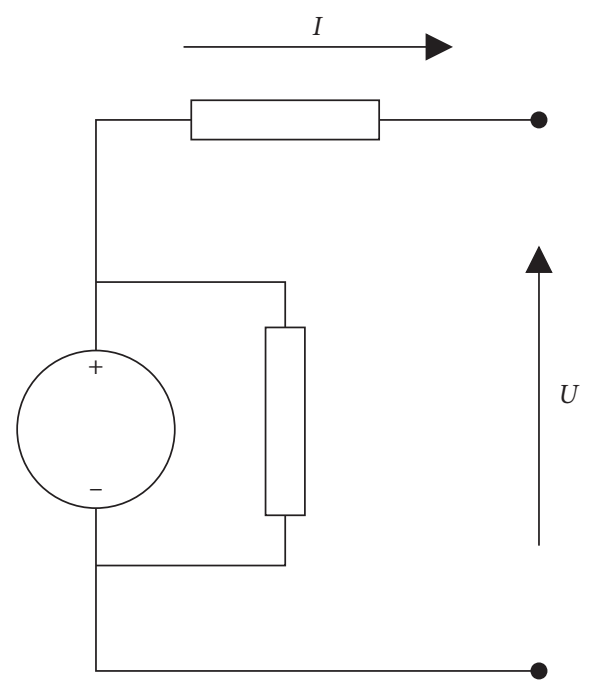

FIGURE 3: Internal-resistance model of the ultracapacitor.

Because the cycle life of an ultracapacitor can reach more than 1 million times, the effect of the life decay can be ignored, and the mathematical model is described as follows:

$$
\left\{\begin{array}{l}
C_{u}=\frac{n_{u 2} C_{\mathrm{uc}}}{n_{u 1}}, \\
R_{u}=\frac{n_{u 1} R_{\mathrm{uc}}}{n_{u 2}}, \\
U_{u}=n_{u 1} U_{\mathrm{uc}}, \\
\mathrm{SOC}_{u}=\frac{U_{\mathrm{uc}}}{U_{\mathrm{un}}}, \\
E_{u}=0.5 n_{u 1} n_{u 2} C_{\mathrm{uc}} U_{\mathrm{un}}^{2} \mathrm{SOC}_{u}^{2},
\end{array}\right.
$$

where $C_{u}, R_{u}$, and $U_{u}$, respectively, represent the capacity, internal resistance, and terminal voltage of the ultracapacitor; $C_{\mathrm{uc}}, R_{\mathrm{uc}}$, and $U_{\mathrm{uc}}$, respectively, represent the capacity, internal resistance, and terminal voltage of the monomer; $n_{u 1}$ and $n_{u 2}$, respectively, represent the number of series monomers and parallel modules in the ultracapacitor; and $U_{\text {un }}$ is the nominal voltage.

\section{Control Strategy and Optimization of HESS}

3.1. Fuzzy Control Strategy. A top-level model of an EV vehicle is shown in Figure 4. There are two energy storage devices in the energy system for EVs, the battery and ultracapacitor. These jointly output power to meet the power demand of the vehicle. Owing to the complexity of the actual operation conditions of the vehicle, unlike a pure EV with a single power supply, the output power of the battery is used to cope with all conditions. The output power of the battery and ultracapacitor should be reasonably distributed by the control strategy of HESS to improve the working efficiency and economy of the vehicle [26].
According to the operation conditions and power demand of the EV, the demand power of the EV is $P_{\text {req }}$, and the output power of the HESS $\left(P_{\mathrm{hy}}\right)$ is composed of the battery output power $\left(P_{\mathrm{bat}}\right)$, ultracapacitor output power $\left(P_{\mathrm{uc}}\right)$, and system loss power $\left(P_{e}\right)$. The mathematical model can be written as

$$
\begin{aligned}
& P_{\text {hy }}=P_{\text {bat }}+P_{\mathrm{uc}}+P_{e}, \\
& P_{\text {req }}=P_{\text {hy }}-P_{e} .
\end{aligned}
$$

Since the energy loss of the system is low and difficult to calculate and the loss is ignored in this study, the output power of the HESS can be calculated as

$$
P_{\text {req }}=P_{\text {hy }}=P_{\text {bat }}+P_{\text {uc }} \text {. }
$$

In the operating process of the vehicle, the output power of the battery pack and ultracapacitor is mainly determined by the SOC of the battery/ultracapacitor and the system demand power. Therefore, energy distribution factors $\left(K_{\text {bat }}\right.$ and $K_{\mathrm{uc}}$ ) are proposed to describe the power output of the battery and capacitor, shown as follows:

$$
\left\{\begin{array}{l}
P_{\mathrm{bat}}=P_{\mathrm{req}} K_{\mathrm{bat}}, \\
P_{\mathrm{uc}}=P_{\mathrm{req}} K_{\mathrm{uc}}, \\
K_{\mathrm{bat}}+K_{\mathrm{uc}}=1 .
\end{array}\right.
$$

Fuzzy control is widely used in various fields. For the management of vehicle energy system, the control method can set different control variables and controlled objects and improve the fuel economy and emissions of the whole vehicle by establishing different fuzzy rules [27-29]. Based on the structure and power requirement of the energy system, the structure of fuzzy control logic is shown in Figure 5. A fuzzy logic control strategy is used to manage the energy transport, and two fuzzy control rules that represent the output power for driving and recovery power for braking are established. The fuzzy logic rule for the output driving energy adopts the form of three inputs and one output. The three inputs are the vehicle demand power $\left(P_{\text {req }}\right)$ and the SOC of the battery $\left(\mathrm{SOC}_{\mathrm{bat}}\right)$ and ultracapacitor $\left(\mathrm{SOC}_{\mathrm{uc}}\right)$. The output is the energy distribution factor $\left(K_{\mathrm{uc}}\right)$. The fuzzy logic rule of braking energy recovery adopts the form of two inputs and one output. The two inputs are the SOCs of the battery $\left(\mathrm{SOC}_{\mathrm{bat}}\right)$ and ultracapacitor $\left(\mathrm{SOC}_{\mathrm{uc}}\right)$, and the output is the energy distribution factor $\left(K_{\mathrm{uc}}\right)$.

The construction form can avoid the frequency of high current output from the battery as much as possible on the premise that the power performance of the vehicle is satisfied. When there is a high energy demand, the ultracapacitor must have enough energy output power. When carrying out braking energy recovery, the ultracapacitor is used for energy recovery.

The Expert Experience Method is used to determine the membership function, and the Gauss, $Z, S$, and Triangle functions are selected as the membership functions to establish the fuzzy control rules of the HESS. The surface view of fuzzy rules is shown in Figure 6. 


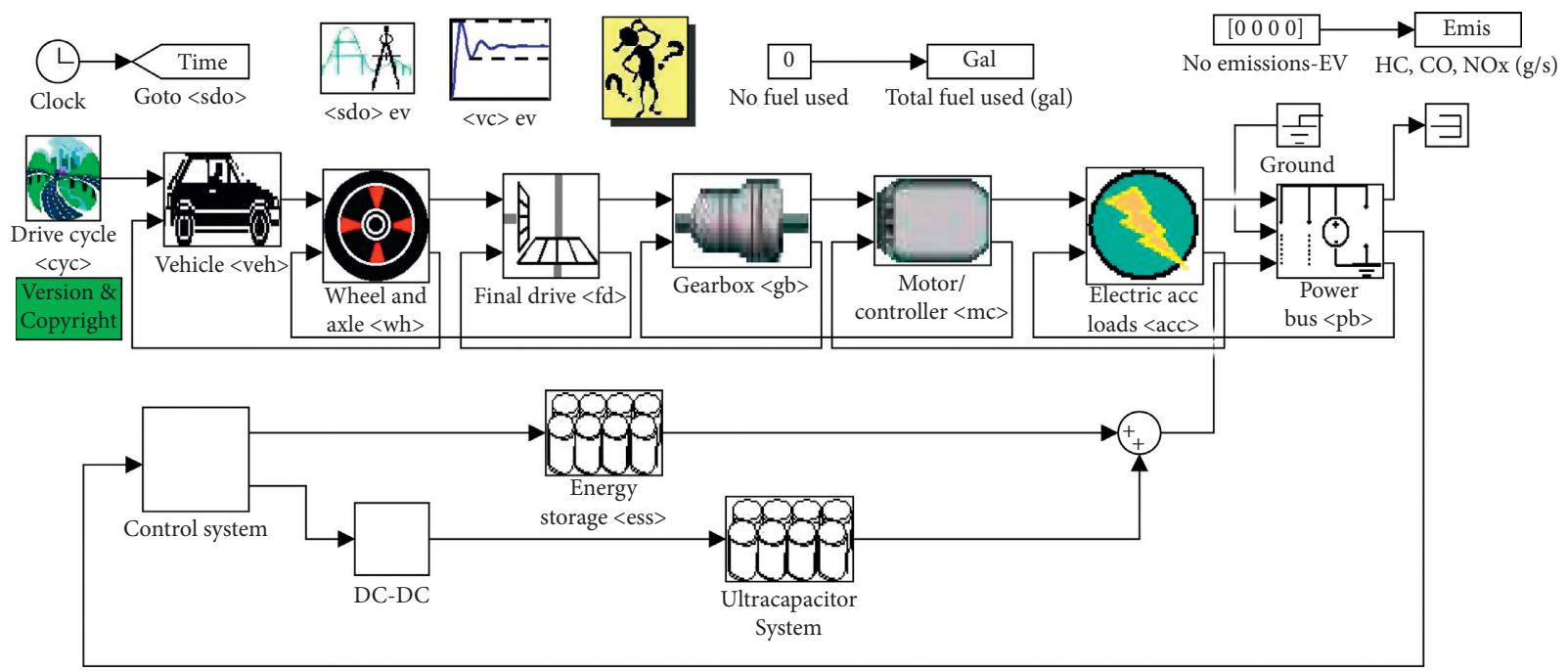

Figure 4: Top-level model of the EV.

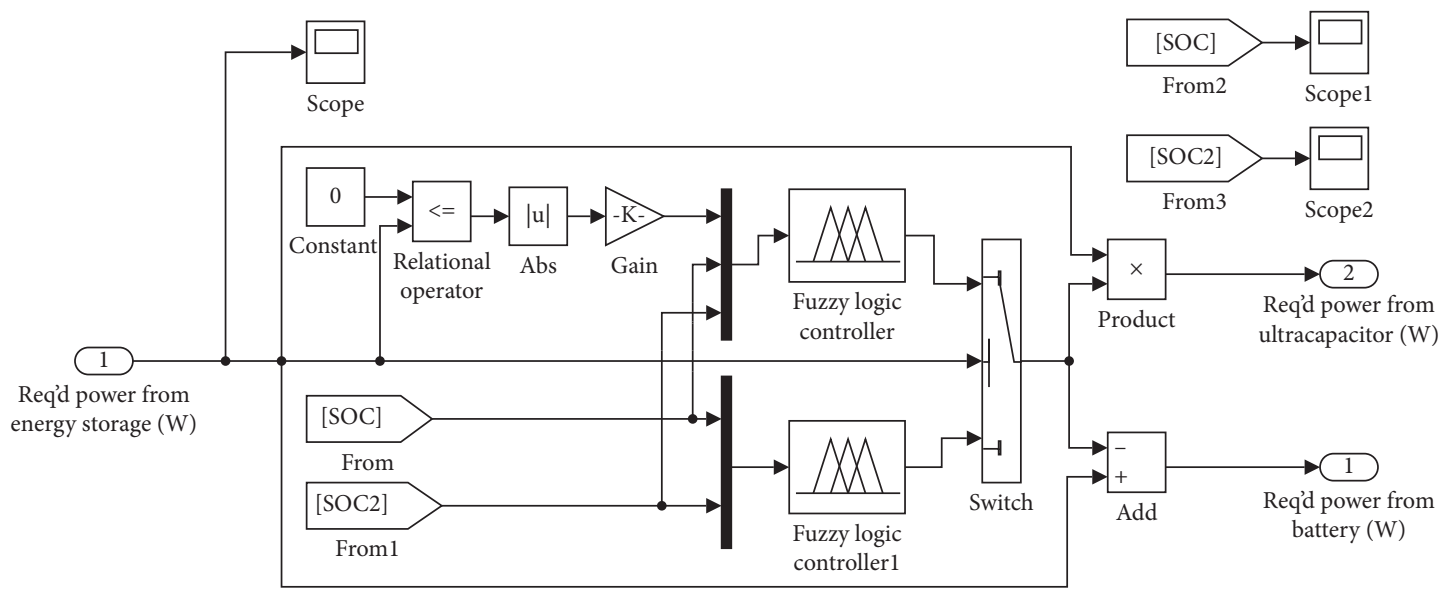

FIgURE 5: Structure of fuzzy logic control.

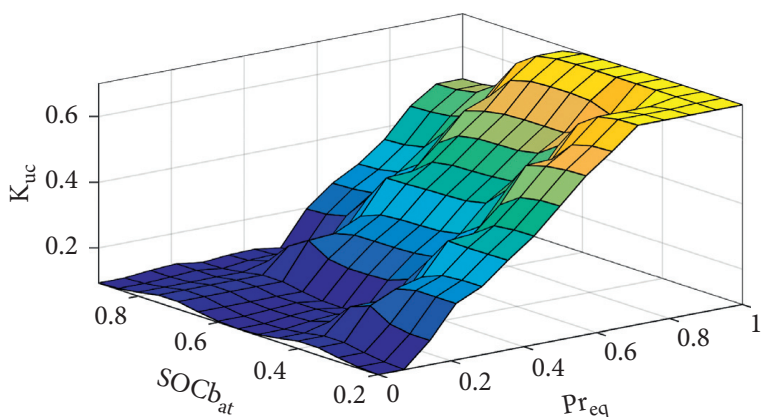

(a)

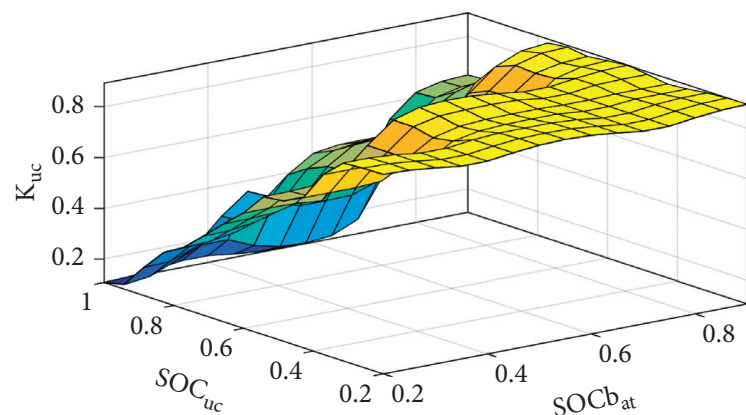

(b)

Figure 6: The surface view of fuzzy rules. (a) Driving. (b) Braking.

3.2. Algorithm Optimization. In order to use the algorithm for optimization, it is necessary to transform the specific problem into a mathematical model and establish the mapping relationship between the value space and coding; that is, the coding is used to represent the problem [30]. Because there are 27 membership functions in the fuzzy controller in this paper, the Gauss, $Z$, and $S$ membership functions need only two variables to determine their 
position and shape, while the Triangle function needs three variables to determine the position and shape of the function. Therefore, 65 parameters are needed to express the value space, as follows:

$$
X=\left(x_{1}^{1}, x_{1}^{2}, \ldots, x_{16}^{1}, x_{16}^{2}, x_{17}^{1}, x_{17}^{2}, x_{17}^{3}, \ldots, x_{27}^{1}, x_{27}^{2}, x_{27}^{3}\right) .
$$

The algorithm is used for optimization, and the mathematical model of the objective function is described as

$$
\min y=f(x) \text {. }
$$

The energy consumption per unit mileage is set as the evaluation standard for the algorithm. It is shown as

$$
f(x)=\text { fitness }=\frac{\text { energy }}{\text { distance }} \text {. }
$$

The energy consumption of the HESS needs to consider the consumption of various components including the battery loss, supercapacitor loss, DC/DC converter loss, line loss, and motor loss in which the battery, capacitor, and DC/ DC converter are the main consumption objects. Other losses are ignored. The mathematical model is shown as follows:

$$
\left\{\begin{array}{l}
\text { energy }=P_{\mathrm{bat}}+P_{\mathrm{uc}}+E_{\mathrm{bat}}^{l}+E_{\mathrm{uc}}^{l}+E_{\mathrm{dc}}^{l}, \\
E_{\mathrm{bat}}^{l}=I_{\mathrm{bat}}^{2}(t) R \\
E_{\mathrm{uc}}^{l}=I_{\mathrm{uc}}^{2}(t) R_{\mathrm{uc}} \\
E_{\mathrm{dc}}^{l}=I_{\text {in }}(t)\left(1-\eta_{\mathrm{dc}}\right),
\end{array}\right.
$$

where $P_{\text {bat }}$ and $P_{\text {uc }}$ are the output power of the battery pack and the ultracapacitor, respectively; $E_{\mathrm{bat}}^{l}, E_{\mathrm{uc}}^{l}$, and $E_{\mathrm{dc}}^{l}$ are the loss of the battery pack, ultracapacitor, and DC/DC converter, respectively; $I_{\text {bat }}$ and $I_{\mathrm{uc}}$ are the working current of the battery pack and the ultracapacitor, respectively; and $\eta_{\text {in }}$ is the input current of the DC/DC converter.

To sum up, the algorithm optimizes the objective function shown as follows:

$$
\text { fitness }=\frac{P_{\mathrm{bat}}+P_{\mathrm{uc}}}{\text { distance }} \text {. }
$$

3.2.1. Genetic Algorithm Optimization. A genetic algorithm (GA) simulates the evolution phenomenon of the Darwinian theory of survival of the fittest in nature and uses the process of survival of the fittest and continuous genetic optimization in the process of evolution to solve the problem and find the optimal solution. All solutions are encoded, and the range of the solution is constantly close to the optimal solution through generations of genetic operations to solve the problem. Based on the evolutionary characteristics of the GA, the inherent properties of the problem are not needed in the process of searching the solution. The ergodicity of the individual enables the algorithm to effectively carry out a global search in the sense of probability, and has better identification accuracy for the entire world [31, 32]. The process of the GA is shown in Figure 7.

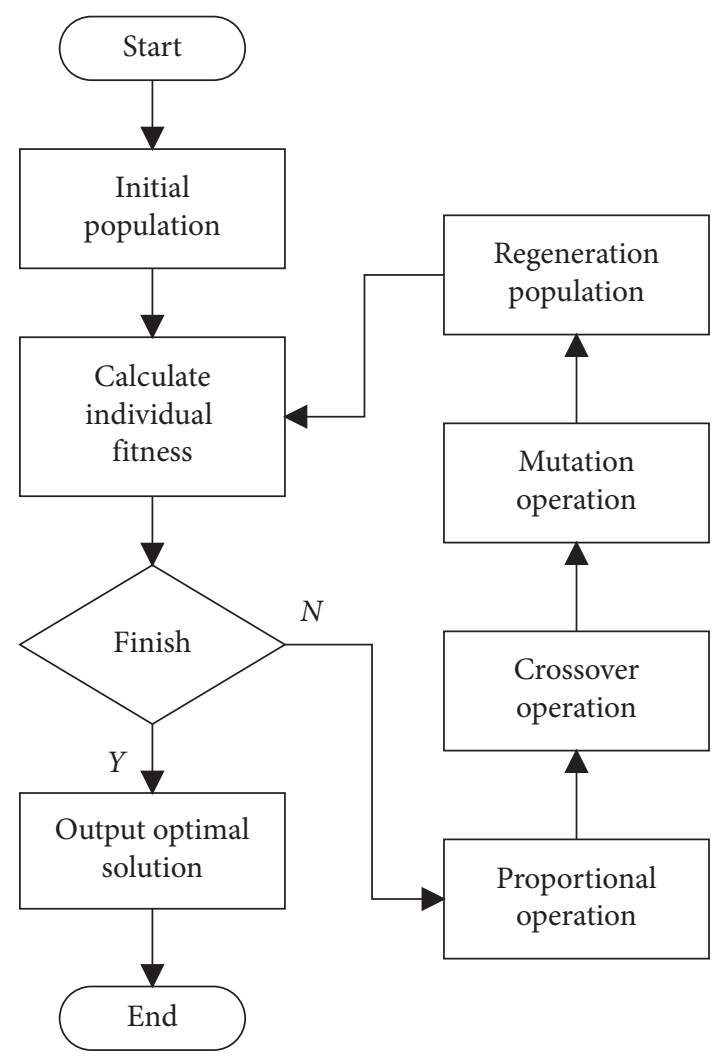

FIgURE 7: Flowchart of GA.

First, the solution to the specific problem is encoded, and the set of corresponding potential solutions is the initial population. Suppose that there are $n$ individuals in an initial population, and the corresponding chromosomes and fitness are shown as

$$
\begin{aligned}
\text { chrom } & =\left[\begin{array}{cc}
x_{1}^{1} & x_{2}^{1} \\
x_{1}^{2} & x_{2}^{2} \\
\ldots & \ldots \\
x_{1}^{n} & x_{2}^{n}
\end{array}\right], \\
\text { fitness } & =\left[f_{1}, f_{2}, f_{3}, \ldots, f_{n}\right] .
\end{aligned}
$$

Then, according to the specific problem, different strategies are used to evaluate the fitness of individuals, and the offspring is selected according to the fitness. Individuals with high fitness are more likely to be selected. New populations are generated through cross recombination and mutation. When they are inherited from the selected algebra or meet the fitness requirements, the individuals with the highest fitness output from the current population are taken as the optimal solution.

In this paper, the control strategy is optimized based on the GA. The steps of building the GA-Fuzzy Control are as follows:

(1) Initialization algorithm: set the evolution algebra to 80 , number of variables to 65 , recombination probability to 0.5 , mutation probability to 0.001 , and generation gap to 0.95 
(2) Initialization population: 65 individuals are randomly generated within the target range as the initial population

(3) Calculate fitness: calculate individual fitness according to the fitness function

(4) Judgment condition: whether the highest fitness of the individual meets the requirements, or whether the evolutionary algebra is terminated

(5) Update the population: select, cross over, and mutate the population to generate a new population, and return to the judgment conditions to continue the evolution process

(6) Save the optimal solution, and establish the GAFuzzy Control strategy embedded in the EV model of MATLAB/Advisor for simulation

3.2.2. Particle Swarm Optimization. PSO is a type of global random search algorithm based on swarm intelligence. It simulates the migration and swarm behavior in the process of bird swarm foraging. When solving specific problems, in the target search space, by combining the individual optimal solution and the group optimal solution, the optimal solution of the target area is searched iteratively [33-36]. A flowchart of the PSO is shown in Figure 8 .

In the $D$-dimensional target search space, the initial population is composed of $n$ particles, where the position and velocity of the $i^{\text {th }}$ particle are $D$-dimensional vectors, shown as follows:

$$
\begin{gathered}
X_{i}=\left(x_{i 1}, x_{i 2}, x_{i 3}, \ldots, x_{i D}\right), \quad i=1,2,3, \ldots, n . \\
V_{i}=\left(v_{i 1}, v_{i 2}, v_{i 3}, \ldots, v_{i D}\right), \quad i=1,2,3, \ldots, i .
\end{gathered}
$$

The optimal positions searched by the $i^{\text {th }}$ particle and the entire particle swarm are the individual extremum and global extremum, respectively, shown as follows:

$$
\begin{aligned}
P_{b} & =\left(p_{i 1}, p_{i 2}, p_{i 3}, \ldots, p_{i D}\right), \quad i=1,2,3, \ldots, n . \\
G_{b} & =\left(p_{g 1}, p_{g 2}, p_{g 3}, \ldots, p_{g D}\right) .
\end{aligned}
$$

After the individual and global extremum are updated, the particle updates its own speed and position according to the current position and speed and the distance from the optimal particle; the update rule is

$$
\begin{aligned}
V_{i d}= & \omega v_{i d}+c_{1} \operatorname{random}(0,1)\left(p_{i d}-x_{i d}\right) \\
& +c_{2} \operatorname{random}(0,1)\left(p_{g d}-x_{i d}\right), \\
x_{i d}= & x_{i d}+v_{i d} .
\end{aligned}
$$

where $\omega$ is the inertia factor (adjusting the global optimization ability and local optimization performance) and $c_{1}$ and $c_{2}$ are acceleration constants, where the former is the individual learning factor of each particle and the latter is the social learning factor of each particle. These are usually set as $c_{1}=c_{2} \in[0,4]$.

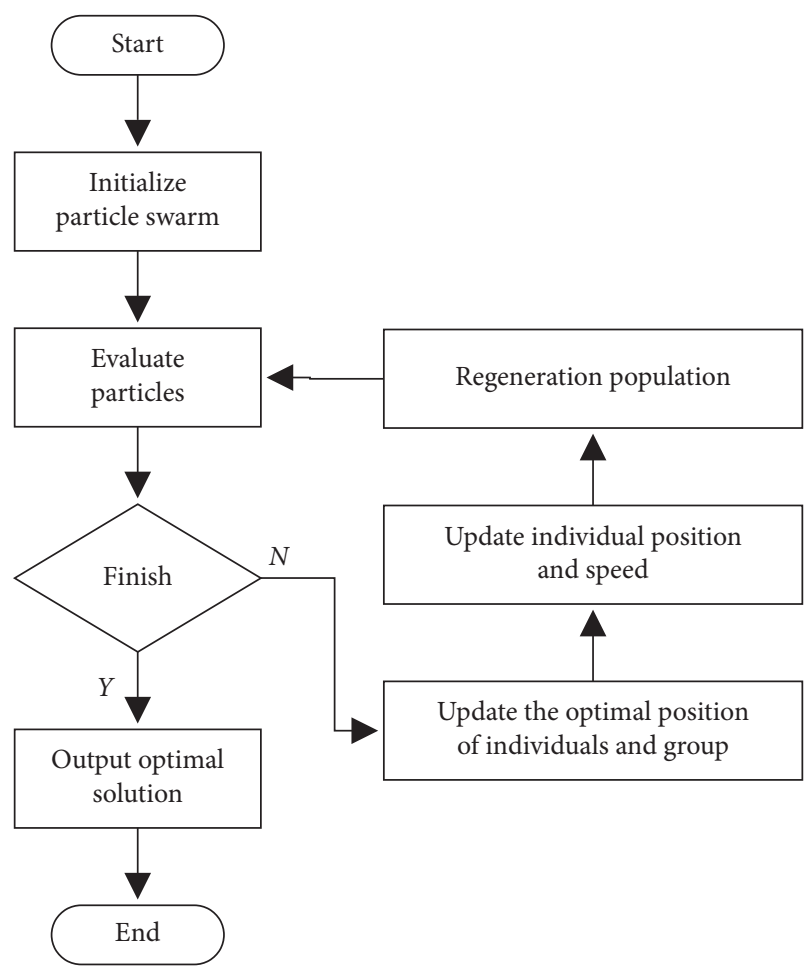

FIGURE 8: Flowchart of PSO.

Based on a PSO algorithm to optimize the control strategy, the steps of building PSO-Fuzzy Control are as follows:

(1) Initialization algorithm: set the maximum number of iterations to 80 , number of particles to 65 , maximum speed to 0.5 , and minimum speed to -0.5

(2) Initialize particle swarm: randomly generate particles with different positions and velocities in the target search space

(3) Evaluate particles: calculate the fitness of particles according to the evaluation criteria

(4) Update the optimum: update the optimal position experienced by particles and groups

(5) Judgment condition: whether the optimal fitness of particles meets the requirements, or whether the iterations are terminated

(6) The optimal solution is saved, and the PSO-Fuzzy Control strategy is embedded into the EV vehicle model of MATLAB/Advisor for simulation

3.3. Dynamic Programming. In order to compare the control performance of GA-Fuzzy Control and PSO-Fuzzy Control more accurately, this paper proposes a dynamic programming (DP) algorithm to calculate the theoretical minimum energy consumption of HESS. DP algorithm is usually used to solve multistage decision-making optimization problems, which are decomposed into subproblems and solved step by step. Because HESS energy management strategy can be considered as a multistage decision-making problem in 
discrete time, the power output of battery and ultracapacitor can be regulated in different stages to obtain the best control performance. Therefore, DP algorithm is suitable for the benchmark evaluation method of HESS energy management strategy [37].

In this paper, the subproblem is to solve the minimum energy consumption of HESS when the initial state is transferred to the current state variable group. In each stage, the solution and optimization are carried out, and finally the minimum energy consumption of HESS in each stage is obtained.

The optimization objective is shown as follows:

$$
E_{\text {con }}=\min \sum_{t=1}\left(E_{b}(t)+E_{u}(t)\right) .
$$

During the optimization process, the ultracapacitor SOC is constrained so that the SOC of ultracapacitor at the end is consistent with that at the initial state. The expression is as follows:

$$
\left\{\begin{array}{l}
I_{\mathrm{bat}, \min } \leq I_{\mathrm{bat}} \leq I_{\mathrm{bat}, \max } \\
I_{\mathrm{uc}, \min } \leq I_{\mathrm{uc}} \leq I_{\mathrm{uc}, \max } \\
0.2 \leq \mathrm{SOC}_{\mathrm{uc}} \leq 1 \\
\mathrm{SOC}_{\mathrm{uc}, \text { initial }}=\mathrm{SOC}_{\mathrm{uc}, \text { end }} \\
4 R_{\mathrm{bat}} P_{\mathrm{uc} \_ \text {ref }} \leq U_{\mathrm{bat}}^{2},
\end{array}\right.
$$

where $P_{\text {uc_ref }}$ is the theoretical output power of the ultracapacitor.

The input variable in the optimization process is $P_{d}$, the state variable is $\mathrm{SOC}_{\mathrm{uc}}$, and the decision variable is $P_{\mathrm{uc} \_ \text {ref }}$. First, the theoretical output power of ultracapacitor is calculated, shown as follows:

$$
\begin{gathered}
E_{\mathrm{uc}}(i, j, k, m)=0.5 \mathrm{CU}_{\mathrm{uc}}^{2}\left(\operatorname{SOC}_{\mathrm{uc}}^{2}(1, m)-\operatorname{SOC}_{\mathrm{uc}}^{2}(1, j)\right), \\
P_{\mathrm{uc} \_ \text {ref }}(i, j, k, m)=E_{\mathrm{uc}}(i, j, k, m)-R_{\mathrm{uc}}\left(\frac{E_{\mathrm{uc}}(i, j, k, m)}{U_{\mathrm{uc}} \operatorname{SOC}_{\mathrm{uc}}(1, m)}\right)^{2} .
\end{gathered}
$$

According to the system demand power and theoretical output power of ultracapacitor, the battery current and energy consumption are calculated:

$$
\begin{aligned}
& I_{\text {bat }}(i, j, k, m)=\frac{\sqrt{U_{\text {bat }}-4 R_{\text {bat }} P_{\text {uc_ref }}(i, j, k, m)}}{2 R_{\text {bat }}}, \\
& E_{\text {bat }}(i, j, k, m)=I_{\text {bat }}(i, j, k, m) U_{\text {bat }} .
\end{aligned}
$$

At the end of calculation, the minimum value can be store in $E_{\text {con_ref }}$ by comparing the energy consumption of each stage:

$$
\left\{\begin{array}{l}
\text { if } E_{\text {con_ref }}(i+1, j, k)>E_{\text {state }}(i, j, k, m)+E_{\text {con_state }}, \\
\text { then } E_{\text {con_ref }}(i+1, j, k)=E_{\text {state }}(i, j, k, m)+E_{\text {con_state }}
\end{array}\right.
$$

After the minimum energy consumption of the system is obtained, the optimal allocation mode can also be obtained through path backward pushing.

\section{Results and Discussion}

In order to confirm the effect of algorithm optimization, the fuzzy control strategy of a HESS optimized by the GA and PSO algorithms is examined. The improved EV model in MATLAB/ Advisor is used for simulations. The following simulation driving cycles are used: UDDS, NEDC, and ChinaCity. A schematic diagram of the operation is shown in Figure 9. The cycle conditions of the three different countries and regions can effectively test the performance of the optimized HESS.

4.1. Analysis of Simulation Results. To evaluate the battery protection performance of the energy management strategy (EMS) optimized by different algorithms, as shown in Figure 10, the battery working currents for different driving cycles are compared. It can be seen that, based on the three conditions, the output current fluctuation of the battery is more stable in the simulation process. From Table 1, in the conditions of UDDS, NEDC, and ChinaCity, the peak current of GA-Fuzzy Control is lower than that of PSO-Fuzzy Control by 35.6001 A, 19.9046 A, and $46.5270 \mathrm{~A}$, respectively.

In general, the economy of the vehicle can be evaluated by examining the fuel economy of the vehicle. As this study is based on an EV, other losses are ignored, and the energy consumption of the HESS is regarded as the economic evaluation standard of the entire vehicle. Figure 11 shows the total energy consumption of two different strategies for simulations in various operating conditions. It can be seen from the figure that, in three driving cycles, the total energy consumption when using GA-Fuzzy Control and PSOFuzzy Control as energy management strategies is lower than that before optimization. This verifies the effectiveness of the algorithm optimization.

Compared with the data in Table 1, in the operating conditions of UDDS, NEDC, and ChinaCity, the total energy consumption of GA-Fuzzy Control decreased by $2.4489 \%$, $9.0604 \%$, and $2.5332 \%$, respectively, compared with that before optimization. The energy consumption of PSO-Fuzzy Control decreased by $1.0859 \%, 0.9659 \%$, and $0.2650 \%$, respectively. The simulation results of the two strategies show that the total energy consumption of the control strategy optimized by the GA is lower. Combined with the comparison results of the working current of the battery, the optimization effect of the GA, in terms of protection of the battery and the battery life stability, is better, which helps save more energy.

Keeping the simulation conditions unchanged, this paper uses the DP algorithm to calculate the theoretical minimum energy consumption of HESS, which is listed in Table 1 for comparison. Compared with the theoretical minimum energy consumption, the simulation results of GA-Fuzzy Control under three drive cycles increased by $0.44 \%, 0.35 \%, 0.52 \%$, respectively. This proves that the control strategy proposed in this paper is approximately the best for the optimization of HESS energy consumption.

4.2. Discussion. The GA and PSO algorithms have many features in common. After the population is randomly initialized, both of them use fitness function to evaluate the 

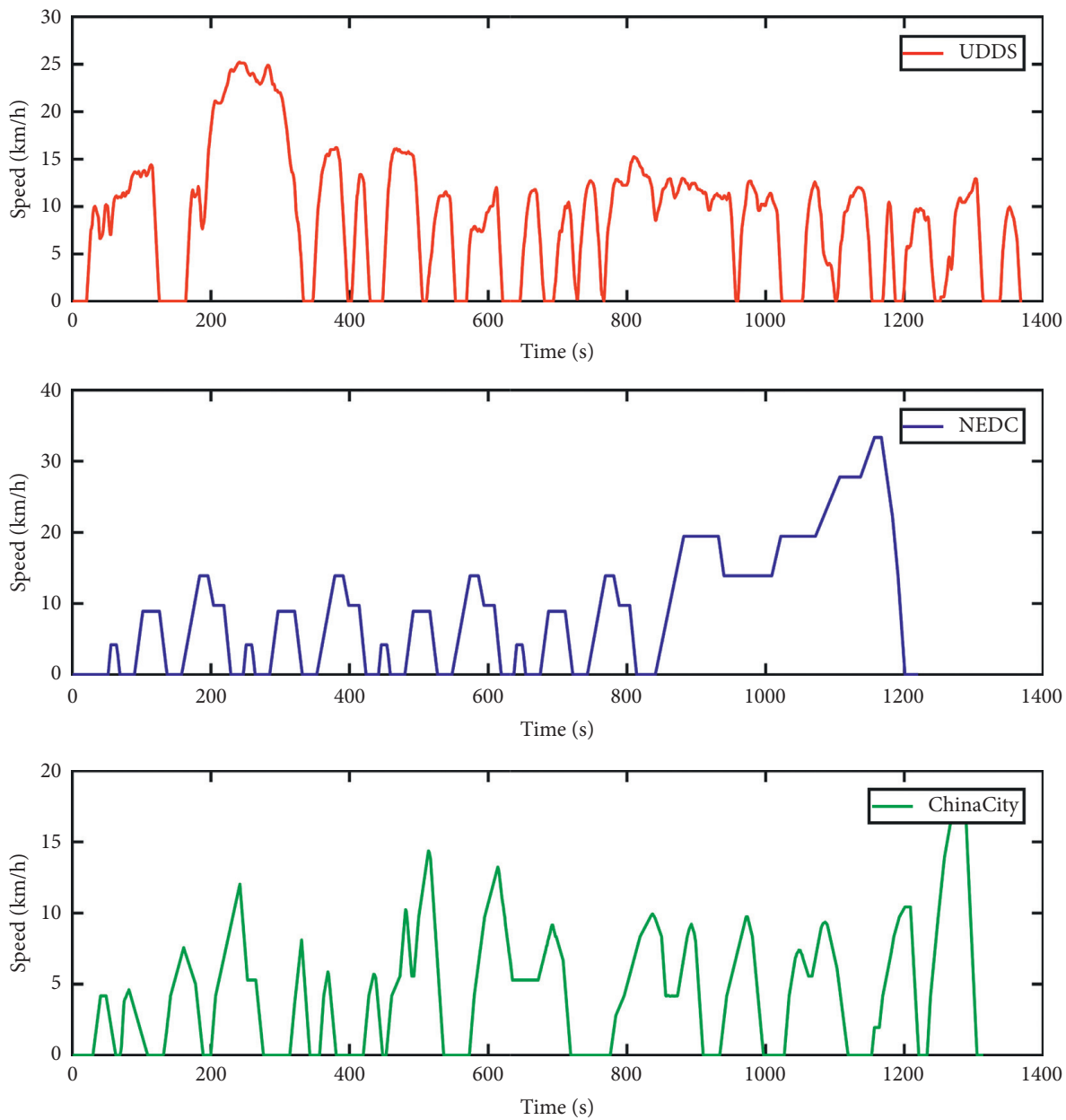

FIGURE 9: Operation diagram of three driving cycles.

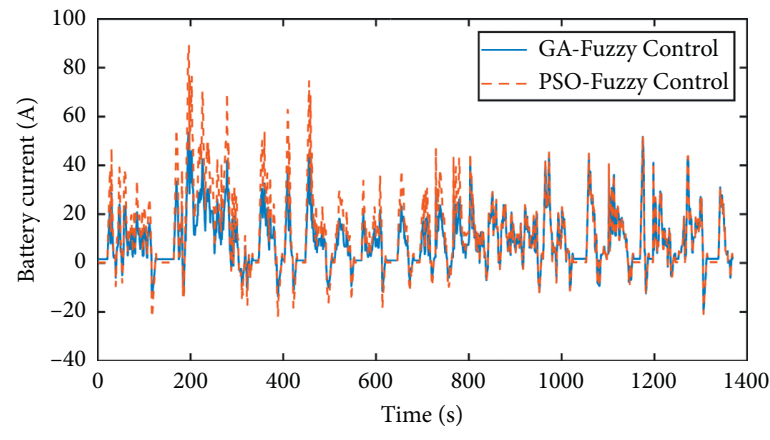

(a)

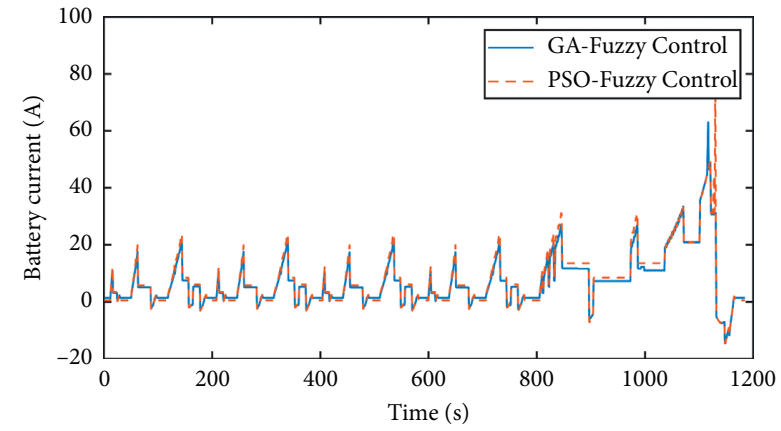

(b)

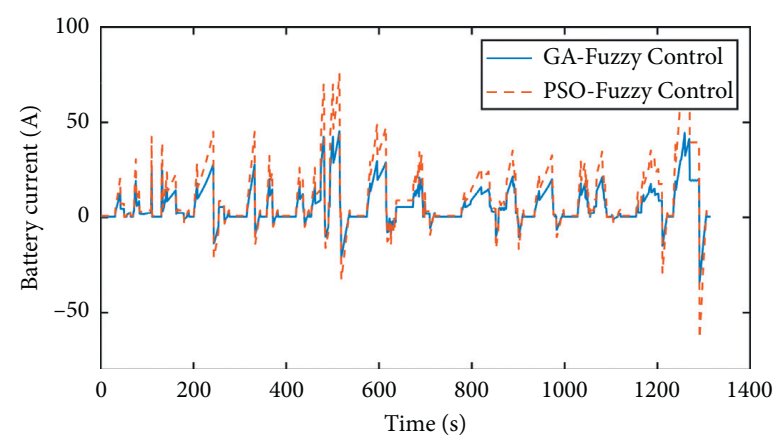

(c)

Figure 10: Battery current for three driving cycles. (a) UDDS, (b) NEDC, and (c) ChinaCity. 
TABLE 1: Parameters of peak current and energy consumption for different scenarios.

\begin{tabular}{|c|c|c|c|}
\hline & & Peak current $(A)$ & Energy consumption $\left(\times 10^{6} \mathrm{~J}\right)$ \\
\hline \multirow{3}{*}{ Fuzzy Control } & UDDS & & 6.7501 \\
\hline & NEDC & & 6.2845 \\
\hline & ChinaCity & & 5.7359 \\
\hline \multirow{3}{*}{ GA-Fuzzy Control } & UDDS & 53.4040 & 6.5848 \\
\hline & NEDC & 62.9496 & 5.7151 \\
\hline & ChinaCity & 45.3493 & 5.5906 \\
\hline \multirow{3}{*}{ PSO-Fuzzy Control } & UDDS & 89.0041 & 6.6768 \\
\hline & NEDC & 82.8542 & 6.2238 \\
\hline & ChinaCity & 91.8763 & 5.7207 \\
\hline \multirow{3}{*}{ DP } & UDDS & & 6.5561 \\
\hline & NEDC & & 5.6954 \\
\hline & ChinaCity & & 5.5619 \\
\hline
\end{tabular}

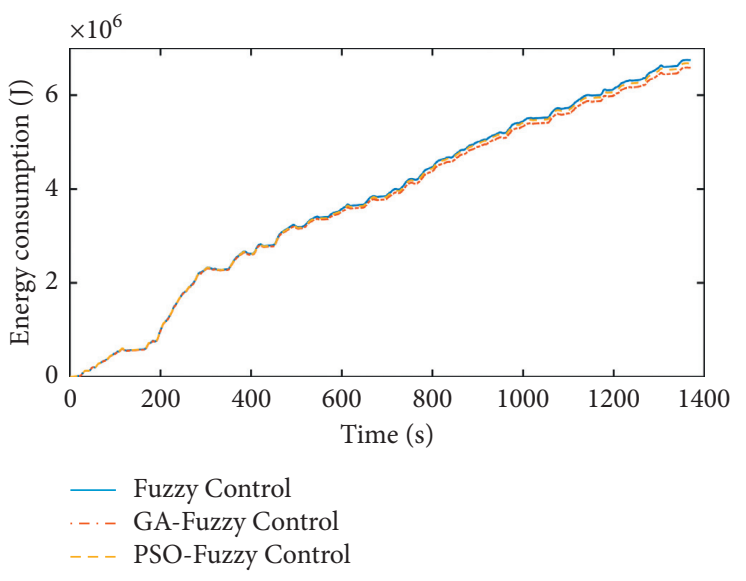

(a)

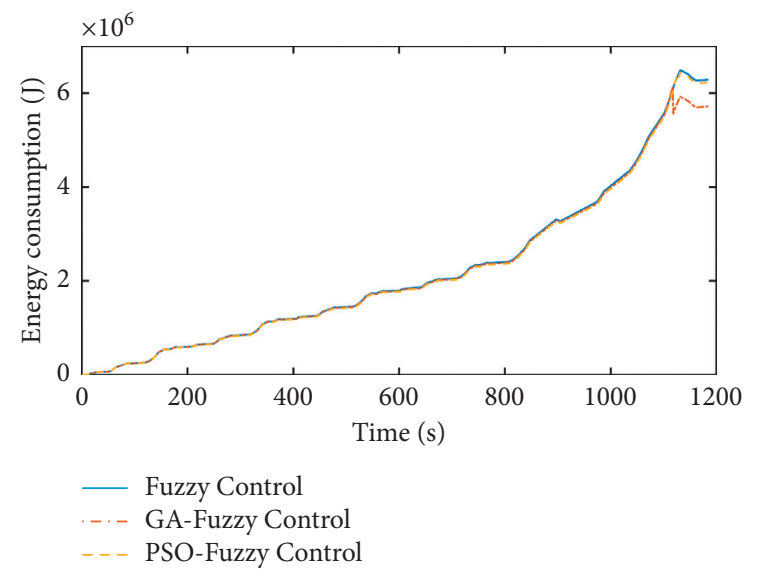

(b)

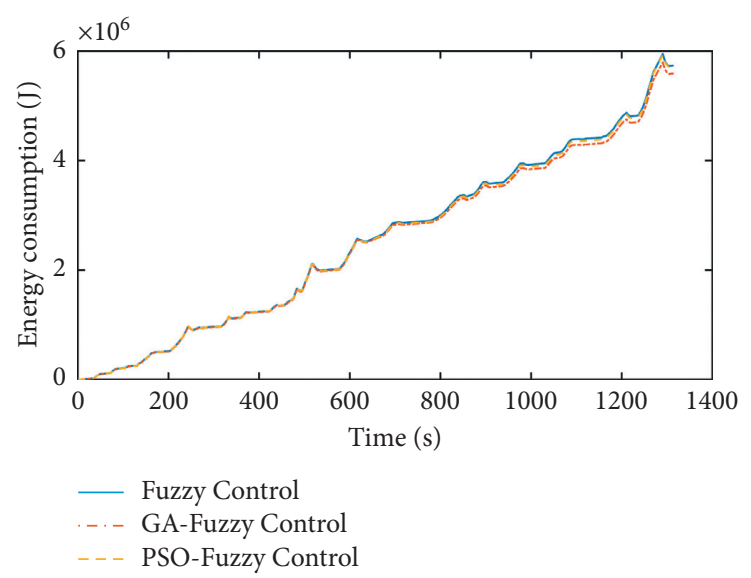

(c)

FIgURE 11: Energy consumption for three driving cycles. (a) UDDS, (b) NEDC, and (c) ChinaCity.

system and search randomly according to the fitness function.

In this paper, based on the control strategy of the hybrid power system of the pure electric vehicle, under the same optimization conditions, the fuzzy rules are optimized by using the GA and PSO. By comparing the control effects of GA-Fuzzy Control and PSO-Fuzzy Control, the accuracy of the two algorithms is evaluated. The results show that the GA is more accurate.

In $[38,39]$, the two algorithms are also used for research. Reference [38] focuses on the optimization of kinetic parameters of biomass pyrolysis. The results show that the PSO based on the three-component parallel reaction mechanism of biomass pyrolysis has the advantages of being closer to the 
global optimal solution and having faster convergence speed than the GA. In [39], two models of algorithms are established and applied to Iran's oil demand forecast. The results show that the demand estimation models of the two algorithms are in good agreement with the observed data, but the PSO model has the best performance.

In the case of binary distribution or discrete distribution of the data in this paper, crossover and mutation operations in the GA are very helpful to find the global optimum, and the effect is better than the gradual approximation of PSO. In $[40,41]$, for global optimization with continuous value, PSO optimization has memory function. It moves to global and local optimal direction in each iteration and can approach the optimal solution faster. It has strong optimization performance and fast optimization speed.

\section{Conclusions}

Based on the characteristics of poor life stability and limited battery life of an EV as a new energy vehicle, this paper studied an EMS and optimized the management strategy to reduce the energy consumption of the HESS and protect the battery life.

(1) Aimed at the semiactive battery load structure of the HESS of a pure electric vehicle, a fuzzy control strategy was selected as the power EMS, and the control framework was constructed based on an EV model in MATLAB/Advisor. The output power ratio of the battery and ultracapacitor was controlled through the vehicle demand power and SOCs of the battery and ultracapacitor to achieve the purpose of optimal management.

(2) The energy consumption per unit mileage was set as the evaluation standard of the algorithm. GA and PSO were used to improve the fuzzy control strategy in the software, establish the GA-Fuzzy Control and PSO-Fuzzy Control strategies, and conduct a simulation based on the operating conditions of UDDS, NEDC, and ChinaCity. The results showed that both algorithms can optimize the energy management and control strategy of the energy system, and the GA had better optimization performance. The GA showed better protection and economy in the simulation to meet the requirements for the endurance of pure electric vehicles equipped with a HESS.

(3) The DP algorithm is used as the benchmark method to calculate the theoretical minimum energy consumption of HESS in this simulation environment. Compared with the simulation results of GA-Fuzzy Control, it is verified that the control strategy proposed in this paper is approximately optimal for the optimization of HESS energy consumption.

(4) Both GA and PSO can optimize fuzzy control strategies, but the time-consuming algorithm is not suitable for real-time optimization. The optimization method used in this article is only applicable when the simulation drive cycle is known in advance.

\section{Data Availability}

The data used to support the findings of this study are available from the corresponding author upon request.

\section{Conflicts of Interest}

The authors declare no potential conflicts of interest with respect to the research, authorship, and/or publication of this article.

\section{Acknowledgments}

This research was funded by the National Natural Science Foundation of China and Natural Science Foundation of Zhejiang Province (Grant Nos. 51741810, 71871078, 71874067, and LGG18E080005).

\section{References}

[1] M. Neenu and S. Muthukumaran, "A battery with ultra capacitor hybrid energy storage system in electric vehicles," in Proceedings of the International Conference on Advances in Engineering, IEEE, Nagapattinam, Tamil Nadu, India, March 2012.

[2] J. Cao and A. Emadi, "A new battery/UltraCapacitor hybrid energy storage system for electric, hybrid, and plug-in hybrid electric vehicles," IEEE Transactions on Power Electronics, vol. 27, no. 1, pp. 122-132, 2012.

[3] S. Lu, K. A. Corzine, and M. Ferdowsi, "A new battery/ ultracapacitor energy storage system design and its motor drive integration for hybrid electric vehicles," IEEE Transactions on Vehicular Technology, vol. 56, no. 4, pp. 1516-1523, 2007.

[4] M. E. Choi and S. W. Seo, "Robust energy management of a battery/supercapacitor hybrid energy storage system in an electric vehicle," in Proceedings of the 2012 IEEE International Electric Vehicle Conference, IEEE, Greenville, SC, USA, March 2012.

[5] G. Wang, P. Yang, and J. Zhang, "Fuzzy optimal control and simulation of battery-ultracapacitor dual-energy source storage system for pure electric vehicle," in Proceedings of the Intelligent Control and Information Processing (ICICIP), IEEE, Dalian, China, August 2010.

[6] O. Erdinc, B. Vural, and M. Uzunoglu, "A wavelet-fuzzy logic based energy management strategy for a fuel cell/battery/ ultra-capacitor hybrid vehicular power system," Journal of Power Sources, vol. 194, no. 1, pp. 369-380, 2009.

[7] M. Michalczuk, B. Ufnalski, and L. Grzesiak, "Fuzzy logic control of a hybrid battery-ultracapacitor energy storage for an urban electric vehicle," in Proceedings of the 2013 Eighth International Conference and Exhibition on Ecological Vehicles and Renewable Energies (EVER), Monte Carlo, Monaco, March 2013.

[8] J. Y. Liang, J. L. Zhang, X. Zhang et al., "Energy management strategy for a parallel hybrid electric vehicle equipped with a battery/ultra-capacitor hybrid energy storage system," Journal of Zhejiang University-Science A (Applied Physics \& Engineering), vol. 14, no. 8, pp. 4-22, 2013.

[9] Q. Wang, S. Du, L. Li et al., "Real time strategy of plug-in hybrid electric bus based on particle swarm optimization," Journal of Mechanical Engineering, vol. 53, no. 4, 2017, in Chinese. 
[10] C. Yang, X. Jiao, L. Li et al., "Research on real-time optimization strategy of plug-in hybrid power for bus applications," Journal of Mechanical Engineering, vol. 51, no. 22, pp. 117-125, 2015, in Chinese.

[11] M. Zandi, A. Payman, J.-P. Martin, S. Pierfederici, B. Davat, and F. Meibody-Tabar, "Energy management of a fuel cell/ supercapacitor/battery power source for electric vehicular applications," IEEE Transactions on Vehicular Technology, vol. 60, no. 2, pp. 433-443, 2011.

[12] M. Hajer, K. Ameni, M. Jean-Philippe, A. Mansour, P. Serge, and B. Faouzi, "Implementation of energy management strategy of hybrid power source for electrical vehicle," Energy Conversion and Management, vol. 195, pp. 830-843, 2019.

[13] L. Ji, Z. Quan, H. Yinglong et al., "Dual-loop online intelligent programming for driver-oriented predict energy management of plug-in hybrid electric vehicles," Applied Energy, vol. 253, p. 113617, 2019.

[14] L. W. Chong, Y. W. Wong, R. K. Rajkumar et al., "An adaptive learning control strategy for standalone PV system with battery-supercapacitor hybrid energy storage system," Journal of Power Sources, vol. 394, 2018.

[15] M. Montazeri-Gh and A. Fotouhi, "Traffic condition recognition using the -means clustering method," Scientia Iranica, vol. 18, no. 4, pp. 930-937, 2011.

[16] A. Fotouhi and M. Montazeri-Gh, "Tehran driving cycle development using the k-means clustering method," Scientia Iranica, vol. 20, no. 2, pp. 286-293, 2013.

[17] M. Montazeri, A. Fotouhi, and A. Naderpour, "Driving segment simulation for determination of the most effective driving features for HEV intelligent control," Vehicle System Dynamics, vol. 50, no. 2, pp. 229-246, 2012.

[18] M. Montazeri-Gh, A. Fotouhi, and A. Naderpour, "Driving patterns clustering based on driving features analysis," Proceedings of the Institution of Mechanical Engineers, Part C: Journal of Mechanical Engineering Science, vol. 225, no. 6, pp. 1301-1317, 2011.

[19] C. Zhang, Research on the Theory of Composite Power Supply Matching and Control for Pure Electric Vehicle, Jilin University, Changchun, China, 2017, in Chinese.

[20] A. Fotouhi, D. J. Auger, K. Propp, S. Longo, and M. Wild, “A review on electric vehicle battery modelling: from Lithiumion toward Lithium-Sulphur," Renewable and Sustainable Energy Reviews, vol. 56, pp. 1008-1021, 2016.

[21] V. H. Johnson, "Battery performance models in ADVISOR," Journal of Power Sources, vol. 110, no. 2, pp. 321-329, 2002.

[22] L. Qian, W. Wu, and H. Zhao, "Simulation analysis of battery model based on advisor software," Computer Simulation, no. 8, pp. 166-168, 2004, in Chinese.

[23] J. Cao, "Battery/ultra-capacitor hybrid energy storage system for electric, hybrid electric and plug-in hybrid electric vehicles," Dissertations \& Theses-Gradworks, vol. 27, no. 1, pp. 122-132, 2010.

[24] I. Urasaki, "Hybrid power source with electric double layer capacitor and battery in electric vehicle," in Proceedings of the International Conference on Electrical Machines \& Systems, IEEE, Incheon, South Korea, October 2010.

[25] Y. Tang and A. Khaligh, "On the feasibility of hybrid Battery/ Ultracapacitor Energy Storage Systems for next generation shipboard power systems," in Proceedings of the Vehicle Power and Propulsion Conference (VPPC), 2010, IEEE, Lille, France, September 2010.

[26] Z. He and Z. Fu, "Fuzzy control strategy for energy management of hybrid electric vehicle," Computer Measurement and Control, vol. 21, no. 12, pp. 3256-3259, 2013, in Chinese.
[27] N. A. Kheir, M. A. Salman, and N. J. Schouten, "Emissions and fuel economy trade-off for hybrid vehicles using fuzzy logic," Mathematics and Computers in Simulation, vol. 66, no. 2-3, pp. 155-172, 2004.

[28] N. J. Schouten, M. A. Salman, and N. A. Kheir, "Fuzzy logic control for parallel hybrid vehicles," IEEE Transactions on Control Systems Technology, vol. 10, no. 3, pp. 460-468, 2002.

[29] N. J. Schouten, M. A. Salman, and N. A. Kheir, "Energy management strategies for parallel hybrid vehicles using fuzzy logic," Control Engineering Practice, vol. 11, no. 2, pp. 171-177, 2003.

[30] X. Song and X. Ren, "Energy management strategy of FSM fuzzy hybrid electric vehicle based on improved artificial bee colony algorithm," Modern Manufacturing Engineering, no. 03, pp. 68-119, 2018, in Chinese.

[31] S. G. Li, S. M. Sharkh, F. C. Walsh, and C. N. Zhang, "Energy and battery management of a plug-in series hybrid electric vehicle using fuzzy logic," IEEE Transactions on Vehicular Technology, vol. 60, no. 8, pp. 3571-3585, 2011.

[32] G. Narges, K. Alibakhsh, T. Ashkan, B. Leyli, and M. Amin, "Optimizing a hybrid wind-PV-battery system using GA-PSO and MOPSO for reducing cost and increasing reliability," Energy, vol. 154, pp. 581-591, 2018.

[33] J. J. Liang and P. N. Suganthan, "Dynamic multi-swarm particle swarm optimizer with local search," in Proceedings of the 2005 IEEE Congress on Evolutionary Computation, IEEE, Hong Kong, China, June 2005.

[34] C. Syuan-Yi, W. Chien-Hsun, H. Yi-Hsuan, and C. Cheng-Ta, "Optimal strategies of energy management integrated with transmission control for a hybrid electric vehicle using dynamic particle swarm optimization," Energy, vol. 160, pp. 154-170, 2018.

[35] N. Bounar, S. Labdai, and A. Boulkroune, "PSO-GSA based fuzzy sliding mode controller for DFIG-based wind turbine," ISA Transactions, vol. 85, pp. 177-188, 2019.

[36] H. Zhang and H. Qing, "Parallel multiagent coordination optimization algorithm: implementation, evaluation, and applications," IEEE Transactions on Automation Science and Engineering, vol. 14, no. 2, pp. 984-995, 2016.

[37] C. Song, F. Zhou, and F. Xiao, "Energy management optimization of composite power supply based on dynamic planning," Journal of Jilin University: Engineering Edition, vol. 47 , no. 188 , pp. 8-14, 2001, in Chinese.

[38] Y. Ding, W. Zhang, L. Yu, and K. Lu, "The accuracy and efficiency of GA and PSO optimization schemes on estimating reaction kinetic parameters of biomass pyrolysis," Energy, vol. 176, no. 1, pp. 582-588, 2019.

[39] E. Assareh, M. A. Behrang, M. R. Assari, and A. Ghanbarzadeh, "Application of PSO (particle swarm optimization) and GA (genetic algorithm) techniques on demand estimation of oil in Iran," Energy, vol. 35, no. 12, pp. 5223-5229, 2010.

[40] H. Zhang, "A discrete-time switched linear model of the particle swarm optimization algorithm," Swarm and Evolutionary Computation, vol. 52, p. 100606, 2020.

[41] N. Kassarwani, J. Ohri, and A. Singh, "Performance analysis of dynamic voltage restorer using improved PSO technique," International Journal of Electronics, vol. 106, no. 2, pp. 212236, 2019. 